\title{
An Overview of the Proposed Mode N System in the Context of Alternative Position, Navigation, and Timing (APNT) Development
}

\author{
Brandon Weaver, Gianluca Zampieri, Okuary Osechas, German Aerospace Center (DLR)
}

\section{BIOGRAPHIES}

Brandon Weaver is a researcher at the German Aerospace Center (DLR) and works on alternative navigation system topics. He joined DLR after completing his Masters in Mechanical Engineering at Tufts University. He also worked at the Charles Stark Draper Laboratory, Inc. on GPS related projects after receiving a Bachelors in Mechanical Engineering from Auburn University.

Gianluca Zampieri received a Master degree in Electronics and Telecommunications Engineering from University of Trento, Italy. In 2019, he joined the Alternative Navigation Systems Group at the Institute for Communication and Navigation of the German Aerospace Center (DLR).

Dr. Okuary Osechas is a researcher with the Institute of Communications and Navigation at DLR. He received a PhD in Electrical Engineering from Tufts University in 2014 and joined DLR in 2015. His current research interests revolve around navigation services for civil aviation.

\begin{abstract}
This paper introduces the Mode $\mathrm{N}$ system concept - a terrestrial navigation system intended for aviation - developed by the air navigation service provider (ANSP) of Germany, Deutsche Flugsicherung. Proposed as a new system, Mode N is presented in the context of other alternative position, navigation, and timing (APNT) systems because it blends aspects of these systems together in its own design. This paper leverages that characteristic by identifying the similarities with other APNT systems and tailoring the large body of APNT work to evaluate Mode N and model the range accuracy for the Mode N signal. This range accuracy model is then used in a basic coverage assessment for Germany.
\end{abstract}

\section{INTRODUCTION}

To accommodate the continued growth of air traffic, Air Navigation Service Providers (ANSPs) are planning and implementing programs to increase the capacity and efficiency of airspace. These programs, which include the Next Generation Air Transportation System (NextGen) led by the Federal Aviation Administration (FAA) and the Single European Sky ATM Research Programme (SESAR) commissioned by the European Union, heavily rely on global navigation satellite systems (GNSS) to enable certain capabilities to reach program goals. While intended to serve as the primary source of position, navigation, and time (PNT) for aviation services going forward, GNSS is vulnerable to sources of interference. For this reason, efforts have been taken to identify and develop an alternative PNT (APNT) system that can maintain capabilities supported by GNSS when a GNSS outage occurs.

The ANSP for Germany, Deutsche Flugsicherung (DFS), have proposed a concept for such a system that they call Mode N [1] [2]. The proposed design leverages current navigation and surveillance technology to provide a completely new solution to navigation. As the current APNT environment is filled with a variety of proposed solutions spanning the entire field of communication, navigation, and surveillance (CNS) technologies, it is useful to describe Mode $\mathrm{N}$ within the context of these other APNT systems. This contextual description serves to highlight the interaction of Mode $\mathrm{N}$ with current aviation systems - an important consideration for any system intended to serve aviation users. Additionally, as the Mode $\mathrm{N}$ design uses similar technological principles as other navigation and surveillance systems, the extensive research performed for APNT can be applied to the Mode $\mathrm{N}$ design to provide a preliminary assessment of its navigation performance over Germany. 
This paper provides an overview and preliminary coverage assessment of the proposed Mode $\mathrm{N}$ concept in the context of other APNT technologies. First, the development of APNT research is summarized, with an emphasis on efforts leveraging current navigation and surveillance infrastructure. The Mode $\mathrm{N}$ system concept is then described, and its designs aspects are characterized in relation to similar systems. Lastly, an estimate of the Mode $\mathrm{N}$ ranging accuracy is developed using assumptions derived from the characterization of Mode $\mathrm{N}$ to previously researched APNT technologies and is applied to a coverage assessment over Germany.

\section{DEVELOPMENT OF APNT}

The current state of aviation navigation can be simplified by acknowledging that GNSS has replaced legacy navigation systems such as DME and VHF omnidirectional range (VOR) beacons as the primary method of navigation for aircraft. The PNT services that GNSS provides enable many capabilities in the airspace which are relied upon by modernization efforts in order to accommodate the expected increase in air traffic in a safe and efficient manner. Due to the vulnerabilities of GNSS as outlined in the 2001 Volpe report [3], it was recognized that an alternative system that could enable the same capabilities as GNSS would be necessary to continue safe and efficient operation of airspace as envisioned if GNSS is unavailable [4].

Proposed APNT solutions are generally sourced from the existing CNS environment. A common strategy is to use an aviationcertified signal not originally intended for navigation, which we have termed repurposed aviation signals (RAS). Other proposals include improving legacy systems, transmitting the ground-computed position to an aircraft, and creating new systems entirely. This section provides an overview of these proposed systems and the current state of the APNT environment. These sources of APNT are summarized in Figure 1 below with explanations of the acronyms to follow.

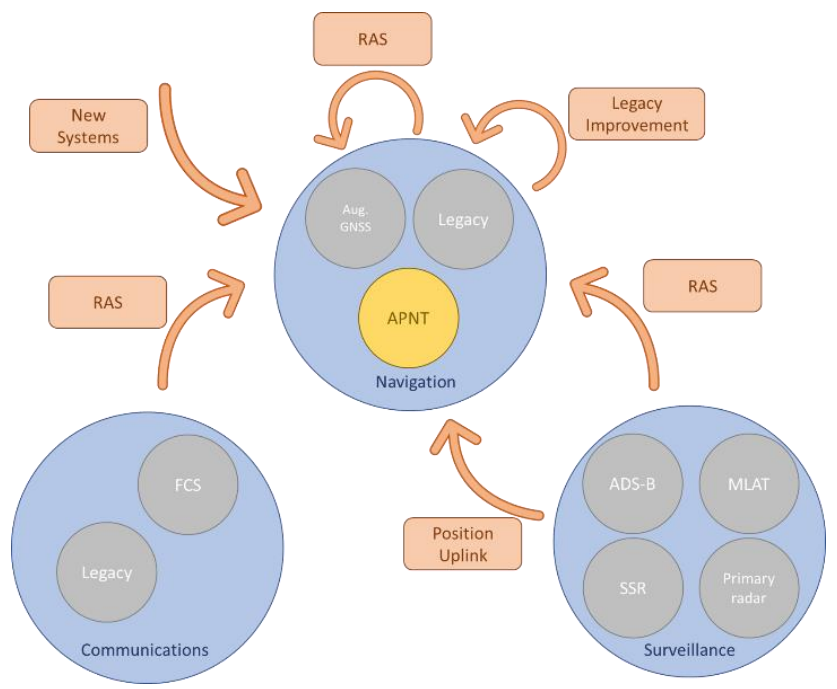

Figure 1 - Sources of APNT for navigation.

A natural candidate for APNT is the use of existing non-GNSS navigation infrastructure. Prior to GNSS, VOR beacons providing beacon-relative heading information and DME navaids supplying two-way range information were the primary navigation infrastructure [5]. Improvement in DME avionics enabled tracking multiple DME stations, providing a DME-only position solution referred to as DME/DME. Adding DME ground stations and upgrading existing hardware to increase accuracy and coverage of DME/DME positioning was therefore an attractive APNT option [6]. Legacy improvement efforts supporting this option included investigating current/expected DME accuracy [7] [8] [9], studying capacity limits [10] [11], and optimizing coverage [6] [7] [12]. Mitigating multipath, a pernicious error resulting from reflected signals, was a large area of focus for DME-based APNT [13] [14] [15] [16]. Enhanced DME (eDME) is a concept for DME that includes additional capabilities such as pseudoranging and would require changes to the DME ground stations [17].

Another option sourced from the existing navigation infrastructure was to use RAS for positioning. One such RAS is that of the DME reply signal to a non-existent aircraft. By triggering DME responses in a desired fashion, aircraft can use these triggered responses for passive ranging without any change to the DME ground stations [18] [19] [20] [21]. The results from a recent flight test for such a system can be found in [22]. The current status for the navigation-based sources of APNT is the expansion of DME coverage in the United States (US) as part of the FAA's NextGen DME program [23]. 
Communication systems for aviation are also undergoing modernization efforts. Future communication systems (FCS) are being developed to provide broadband communication capability between aircraft and controllers. One such system currently undergoing standardization by the International Civil Aviation Organization (ICAO) is the L-band Digital Aeronautics Communications System (LDACS) [24]. The LDACS transmitters provide signals that can be used for navigation (i.e. RAS) with the results from a flight test evaluating LDACS RAS-based navigation presented in [25]. The use of LDACS for navigation is an ongoing effort.

Surveillance is the domain of ground-based systems that determine the position of remote objects and is fundamental to allowing safe spacing of aircraft. Its origins reside in the development of primary radar, which was then complemented with secondary surveillance radar (SSR). Both primary radar and SSR use a rotating antenna to measure range and bearing to determine the bearing of the remote objects and tend to be clustered around airports, limiting its area of coverage. To expand coverage in challenging terrain where primary/SSR is difficult to install, a technique known as multilateration is used where a surveillance ground system can receive a signal from an aircraft and determine its position by comparing the time of arrival (TOA) of the signal between its ground stations [26]. These systems were considered as a source of APNT by providing the aircraft position computed on the ground back to the aircraft via data uplink, but timely authentication and integrity concerns have stalled this approach in the US [27] [28].

\section{Surveillance RAS for APNT}

The other branch of surveillance-sourced APNT is by using RAS and is very relevant to the design of Mode N. The system providing many of the RAS for navigation is ADS-B. With this service, an aircraft broadcasts its GNSS-derived position (ADSB Out) to ground-based stations and any aircraft capable of receiving ADS-B transmissions. ADS-B is an important part of airspace modernization strategies and is mandated for aircraft operating in most U.S. airspace as of Jan 2020 according to CFR 91.225 and 91.227, with European mandates to take effect late 2020. The ADS-B ground stations, referred to as ground-based transceivers (GBT) or radio stations (RS), collect ADS-B Out messages for use by air traffic operators. These RS also provide their own transmissions for use by aircraft that can receive ADS-B broadcasts (ADS-B In capability) and include weather information, nearby air traffic, etc.

ADS-B can use different protocols to transmit its signals. The Mode S (S for Selective) protocol was designed to allow SSR ground stations to selectively interrogate aircraft in its coverage area, reducing congestion on the reply frequency [29]. The Mode S reply format consists of a four-pulse preamble and a data block containing either 56 or 112 information bits for the aircraft to provide information that is dependent on the interrogation received [30]. Mode $S$ is internationally standardized, and an extended format known as Mode S Extended Squitter was adopted for Automatic Dependent Surveillance Broadcast (ADSB) services. Mode S Extended Squitter or 1090ES (as its transmitted exclusively on $1090 \mathrm{MHz}$ ) is also used by the ADS-B RS that re-broadcast ADS-B Out (ADS-R) and provide traffic information services (TIS-B) to nearby aircraft with ADS-B In capability.

Another protocol, used in the US, is the Universal Access Transceiver (UAT) format. Like 1090ES, UAT is used by certain aircraft to transmit their ADS-B Out messages. Similarly, ADS-B RS transmit TIS-B and ADS-R messages with the UAT protocol but also include additional information that it transmits with the Flight Information Service - Broadcast (FIS-B). UAT signals are transmitted in the US on an unused DME channel frequency of $978 \mathrm{MHz}$. Figure 2 summarizes the relationship between these surveillance signals and the services that use them.

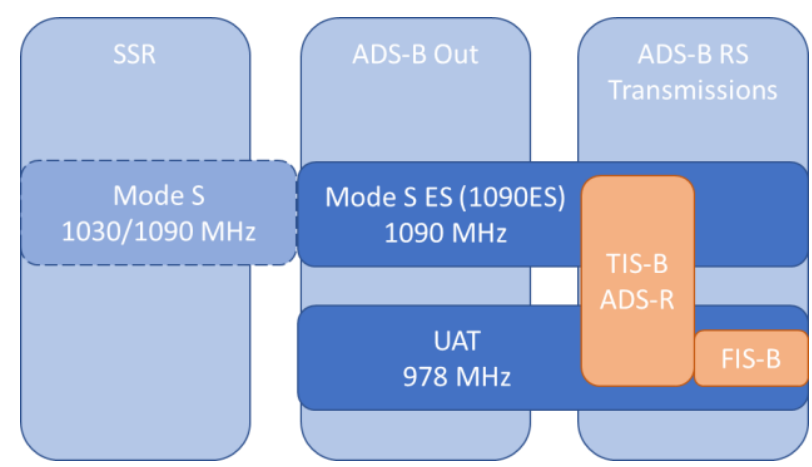

Figure 2 - Services using Mode S and UAT signal formats. 
Research investigating the ground-transmitted (ADS-B RS) 1090ES and UAT signals for ranging measurements greatly supports the assessment of Mode $\mathrm{N}$ presented here, as the Mode $\mathrm{N}$ system operates on a similar basis with a signal that blends characteristics of 1090ES and UAT together.

\section{MODE N OVERVIEW}

Mode $\mathrm{N}$ ( $\mathrm{N}$ for Navigation) is a passive ranging system concept from DFS that seeks to provide APNT while reducing the spectrum congestion caused by existing aeronautical navigation and surveillance systems [1]. The design includes the possibility for two-way and air-to-air ranging, but this overview focuses on the preferred passive mode of operation. It is designed around the Mode S format, which as mentioned is used for SSR and ADS-B services. Despite early references to a "SSR/N" system, Mode N is not a new SSR mode but rather a new navigation system [31] [2].

The basic concept is Mode $\mathrm{N}$ ground stations would transmit signals on a single frequency that include ground station ID/coordinates, allowing aircraft with Mode $\mathrm{N}$ avionics to receive those signals and determine its position in a similar manner to GNSS. As a single frequency is desired to minimize spectrum usage, the ground stations would space their transmissions apart in time to avoid intersystem interference. This scheme, known as time division multiple access (TDMA), would require information within the signal message on the scheduled time a ground station transmits, which the Mode $\mathrm{N}$ format allows.

Because Mode N shares many design aspects with Mode S, DME, and other surveillance RAS, it is able to leverage previous APNT work for the benefit of its own analysis. Therefore, the overview of the design is described here relative to other APNT systems, as this is the basis of the preliminary performance assessment presented in a later section.

\section{The Mode N signal}

The Mode $\mathrm{N}$ design proposes using the Mode $\mathrm{S}$ downlink signal format as the basis for its ranging signal to be used by the aircraft for passive position determination, with some key differences. The frequency channel on $1090 \mathrm{MHz}$ is too congested to accommodate more signals, thus the first difference is that Mode $\mathrm{N}$ intends to transmit on a different frequency. While the channel selection is still ongoing, unused DME channels have been identified as options for frequency allocation. The second difference is the message content. As the Mode $\mathrm{S}$ downlink format transmits mainly aircraft specific information, Mode $\mathrm{N}$ ground transmitters would instead populate their messages with information needed for passive ranging: ground station coordinates and time of transmission (TOT). The study of 1090ES messages (which also contain aircraft-specific information despite being transmitted by ground stations) as RAS required some special techniques to first identify which station was transmitting the message. The TOT is not present in 1090ES signals, but more importantly the time of transmission is not synchronized to any consistent reference. Aside from transmission frequency and message content, the Mode $\mathrm{N}$ signal design follows the Mode $\mathrm{S}$ downlink format (modulation, pulse shape, etc.).

The Mode N signal also shares some aspects with the UAT signal, particularly the FIS-B segment. First, UAT is also transmitted in the US on an unused DME channel. The FIS-B message, which provides weather information, transmits the ground station coordinates and information that can be used to estimate the TOT. Specifically, UAT messages are synced to UTC time, and each ADS-B RS has a designated time slot within a one second interval where it transmits its FIS-B message. This time slot is included in the message, and can be used to determine the TOT of the signal. Mode $\mathrm{N}$ is designed to work in this exact manner, minus the weather information. One crucial difference between UAT and the Mode $\mathrm{N}$ design is the type of modulation. Like Mode S, Mode N proposes using pulse-position-modulation (PPM) or on-off-keying (OOK). The resulting wider bandwidth estimated to be less than $4.6 \mathrm{MHz}$ at $-3 \mathrm{~dB}[1]$ - has better resistance to multipath, whereas UAT is frequency modulated to maintain a narrow bandwidth to avoid interference with DME and is more susceptible to multipath [32]. This research on UAT signals for pseudoranging capability also determined a higher update rate than one second would be necessary for navigation, an important consideration for the final Mode $\mathrm{N}$ design.

\section{Ground Infrastructure}

The Mode N design, while based on RAS from surveillance, requires new ground stations to transmit the Mode $\mathrm{N}$ signal. The requirements for the ground stations are that they provide adequate coverage to meet the requirements of an APNT system and that they are sufficiently synchronized in time. An initial time synchronization scheme outlined in [1] is the use of an RF network consisting of the ground stations themselves, which requires radio line-of-sight of stations throughout the network. DFS performed a study and found that additional time beacon stations would be necessary to maintain this RF time network even though navigation coverage was provided using existing DME sites as hypothetical Mode N stations. Since these aspects 
of the design are still developing, the preliminary assessment presented later assumes a network layout and time synchronization tolerance. As the Mode N design blends various CNS principles, a natural baseline design for the ground station locations are existing DME and surveillance sites in Germany. Using these locations for the ground stations enables computation of a horizontal dilution of precision (HDOP) at discrete locations throughout Germany. The assumed time synchronization is discussed further when developing a model of the Mode N ranging accuracy.

\section{Avionics}

An interesting aspect of the Mode $\mathrm{N}$ design is its proposed avionics unit. The Mode $\mathrm{N}$ avionics must be capable of receiving Mode $\mathrm{N}$ messages, which it can do with the existing DME antennas on aircraft. The Mode $\mathrm{N}$ avionics unit must then decode the messages for position determination. Its active mode for two-way and air-to-air ranging would require the Mode $\mathrm{N}$ avionics to transmit Mode $\mathrm{N}$ messages, again using the existing DME antenna.

Recognizing the continuing investment in the DME network by multiple countries, the Mode $\mathrm{N}$ avionics sensor is essentially built around a fully functional DME unit [1]. This is intended to provide a seamless transition as Mode $\mathrm{N}$ stations are brought online. The design of the avionics has little effect on the coverage assessment, aside from guaranteeing a minimum level of performance based on the current DME network, but is an important part of the implementation strategy. Furthermore, this blend of avionics was also proposed in [33] for a unit that was compatible with DME and ADS-B (1090ES and UAT) signals.

\section{PRELIMINARY COVERAGE ASSESSMENT}

Preliminary coverage assessments are a typical method to determine the feasibility of a proposed system to provide the required level of performance over a given area [12] [7]. A simple method of characterizing the position performance is in terms of the linear relationship between range error and the dilution of position (DOP), where the range errors are assumed to be zero-mean, uncorrelated, and have identical distributions [34].

$$
\sigma_{\text {pos }}=H D O P * \sigma_{\text {range }}
$$

As the aircraft is assumed to have additional sensors for determining its altitude, the horizontal DOP (HDOP) is commonly used to characterize the expected horizontal position performance. The standard deviation of range error and horizontal position error are represented by $\sigma_{\text {range }}$ and $\sigma_{\text {pos }}$, respectively.

With range measurements, HDOP is a function of the transmitter geometry available to an aircraft at a given point. It is a straightforward computation to perform for a grid of points over the area of interest. The HDOP computation does depend on the type of range measurement, so passive (pseudo) range, two-way range, and TDOA measurements all have their corresponding DOP computation. Determining a model for the range error is less straightforward, and assessing the coverage potential of Mode $\mathrm{N}$ requires an estimation of the expected range error.

This section is organized as follows. First, a model for Mode N range accuracy is derived by tailoring the existing APNT body of work to evaluate individual aspects of the Mode $\mathrm{N}$ design that affect the ranging accuracy. Specifically, the research aimed at improving DME performance and at using existing ground-based surveillance signals for ranging is heavily leveraged to predict a level of accuracy for the Mode $\mathrm{N}$ signal. Next, the derived model of range accuracy is used with a baseline ground station network to assess the feasibility of Mode $\mathrm{N}$ as an APNT system.

\section{Modeling Mode N range accuracy}

As Mode $\mathrm{N}$ is not an existing system, abundant quantities of real measurements are unavailable for empirically characterizing the range performance. Since Mode N is heavily based on the Mode S signal format and functions similarly to the DME and UAT signals, which all currently exist and have been measured extensively, research investigating those signals can help derive the model for the Mode $\mathrm{N}$ range performance.

An alternate approach is to reference the standards for a specified performance level. For example, [30] specifies that the Airborne Collision Avoidance System (ACAS) avoidance logic use a zero-mean normal distribution range error model with a standard deviation of $50 \mathrm{ft}$, or about $15 \mathrm{~m}$. As ACAS also uses the Mode $\mathrm{S}$ signal format, this appears to be a reasonable source for the Mode $\mathrm{N}$ range error. However, since ACAS is an airborne two-way surveillance method, it does not exactly translate to a ground-based passive TDOA system such as Mode N. The $15 \mathrm{~m}$ standard deviation is still useful as it provides a check on the 
estimated Mode $\mathrm{N}$ accuracy. Other specifications suffer from similar drawbacks, in that Mode $\mathrm{N}$ does not directly apply to any single system. Thus, the blended approach using previous APNT research is chosen.

To begin deriving the expected range error, the fundamental measurement for the passive ranging mode of Mode $\mathrm{N}$ is presented [1]. Mode $\mathrm{N}$ measures the time difference of arrival (TDOA) between ground stations $i$ and $j$ as shown below.

$$
T D O A_{i j}=\left(t_{o a}^{i}-t_{o t}^{i}-t_{s l o t}^{i}\right)-\left(t_{o a}^{j}-t_{o t}^{j}-t_{s l o t}^{j}\right)
$$

where $t_{o a}^{i}, t_{o t}^{i}, t_{\text {slot }}^{i}$ is the TOA, the system TOT, and designated time slot of signal $i$, respectively. This measurement cancels out the offset of the avionics clock relative to the ground stations assuming a constant offset between reception of signals $i$ and $j$. The $t_{o a}^{i}$ is the avionics clock time referenced to some time-mark of the signal. The $t_{\text {slot }}^{i}$ is provided in the data message, and the $t_{o t}$ of signals $i$ and $j$ are only cancelled if the ground stations are perfectly synchronized. This measurement is in seconds, and is translated to a range difference by using the speed of radio signal propagation in a vacuum, $c$.

$$
\begin{gathered}
\Delta \rho_{i j}=\left(T D O A_{i j}\right) c \\
\Delta \rho_{i j}=\rho_{i}-\rho_{j}
\end{gathered}
$$

Now it is easier to see the relationship between the TDOA measurements and the range accuracy. It is the errors present in $\rho_{i}$ that are referred to collectively as the range error and that are characterized by a standard deviation $\sigma_{\text {range }}$. This paper will describe errors in terms of both range error and TDOA error. The relationship between these errors can be derived from the variance properties of independent random variables, which we have assumed the range/TDOA errors to be.

$$
\begin{aligned}
& \sigma_{\Delta \rho}^{2}=\operatorname{Var}\left(\delta \rho_{i}-\delta \rho_{j}\right) \\
& \sigma_{\Delta \rho}^{2}=\operatorname{Var}\left(\delta \rho_{i}\right)+\operatorname{Var}\left(\delta \rho_{j}\right)=\sigma_{\rho, i}^{2}+\sigma_{\rho, j}^{2} \\
& \text { if } \sigma_{\rho, i}=\sigma_{\rho, j}=\sigma_{\text {range }} \\
& \sigma_{\Delta \rho}=\sqrt{2} \sigma_{\text {range }}
\end{aligned}
$$

The link between range and TDOA measurements is established as most of the APNT research evaluates two-way or passive range performance, so for consistency the derivation of expected Mode $\mathrm{N}$ accuracy is in terms of range.

Looking at (2), errors can be present in the TOA measurement, synchronization of the nominal TOT of the signals, and parsing of the time slot data field. The TOA measurement can have errors by inaccurate determination of the actual TOA due to noise or multipath and by the actual TOA differing from the nominal arrival time of the signal due to atmospheric delay. For terrestrial systems, propagation errors are considered to be dominated by multipath, so atmospheric affects are not considered in this paper. Time synchronization errors are very important to the ranging accuracy, but it is assumed the time slot data field is parsed accurately. Other sources of error, such as inaccurate ground station coordinates, can affect the position error but have no effect on the range error. Additionally, the error originating from the change in aircraft position between reception of signals $i$ and $j$ is not considered in this paper. The model of range accuracy can then be expressed as the root-sum-square (RSS) of the dominant individual error components.

$$
\sigma_{\text {range }}^{2}=\sigma_{\text {noise }}^{2}+\sigma_{\text {multipath }}^{2}+\sigma_{\text {synch }}^{2}
$$

Each error component can now be studied in isolation, selecting the applicable APNT research to leverage based on the Mode $\mathrm{N}$ design aspect that most corresponds with that error.

\section{Noise in TOA determination}

TOA determination refers to correlating some aspect of a received signal to a time measured by a clock. This section refers to errors in determining TOA based on characteristics of the signal itself. Determining the TOA of a signal depends on the type of signal received: for pulsed signals, such as DME and 1090ES, referencing the half-amplitude of a received pulse to a clock is a standard method; for frequency-modulated signals such as UAT, correlation of the signal with a replica is a common technique. The accuracy of the measurement depends on the signal-to-noise ratio (SNR), with noisier signals introducing more error, and generation of the waveform itself.

Since the Mode $\mathrm{N}$ design also uses a pulsed signal, the evaluation of DME (specifically, DME/N) ranging performance is the starting point for estimating the TOA noise error. In [13], Monte Carlo simulations on the effect of noise for the half-amplitude method for a signal with a SNR of $26 \mathrm{~dB}$ resulted in noise errors with a standard deviation $(1 \sigma)$ of $10 \mathrm{~m}$. Part of the APNT effort was evaluating current DME performance, as it was thought it exceeded the specified performance in standards. A bottom-up analysis of DME accuracy was presented in [9] that separated the performance into its main error components based 
on collected DME measurements, with a budgeted TOA error of $10 \mathrm{~m}, 2 \sigma$. Another study found that current (based on data presented in [8]) DME performance allowed a budgeted TOA error of $15 \mathrm{~m}, 2 \sigma$. These results are summarized in Table 1 below.

Table 1 - DME TOA error values

\begin{tabular}{|l|c|c|c|}
\hline Source: & [13], simulated & [9], measured & [15], measured \\
\hline TOA error $(1 \sigma):$ & $10 \mathrm{~m}$ & $5 \mathrm{~m}$ & $7.5 \mathrm{~m}$ \\
\hline
\end{tabular}

For the Mode $\mathrm{N}$ error model, the $7.5 \mathrm{~m}$ error from [15] is an attractive option to choose as it is the average of the other two sources and is the most recent. This value is a conservative estimate of the TOA accuracy for Mode N because the Mode N/S pulse shape is narrower than the DME pulse with a greater bandwidth, improving theoretical accuracy [13] [15]. For the preliminary coverage assessment, a conservative estimate is desired as the actual TOA accuracy will vary over an area depending on transmitter distance - which impacts the level of signal noise. Note that the DME TOA errors are not divided by two as is done for the total DME error as they apply to a one-way TOA measurement.

\section{Multipath}

Multipath is a difficult error to model, as it can result in very large errors in highly localized areas but be nonexistent the rest of the time. A simple, but conservative, model is desired for the preliminary coverage assessment. To estimate the multipath error of a Mode N signal, the research investigating multipath for DME and 1090ES signals is invoked.

Beginning again with DME, the multipath envelope for DME as a function of the delay between the direct and reflected signal is a useful starting point. The analysis in [9] used this envelope for a reflected signal with half the direct signal amplitude and found a maximum multipath error of about $80 \mathrm{~m}$. The same maximum error was found in [15], which also simulated multipath for a range of parameters such as multipath to direct signal amplitude ratio, phase angle difference, and the multipath delay. The root-mean-square (RMS) of the resulting measurement error was $40.6 \mathrm{~m}$ after white noise corresponding to a $30 \mathrm{~dB}$ SNR was added. An error budget for current DME performance is also given in [15], and lists the propagation (i.e. multipath) $2 \sigma$ error to the aircraft as $36 \mathrm{~m}$. Likewise, an error budget in [9] allocated a "reasonable" $40 \mathrm{~m}, 2 \sigma$, to propagation for a potential DME system that would meet a desired accuracy.

While the multipath performance for DME is useful to reference, the 1090ES efforts are the main source used to derive a Mode $\mathrm{N}$ estimate as the signals differ only in frequency (while remaining in the L-band) and message content. The effects of multipath were studied for 1090ES signals in [35] with TOA being determined by correlation of the four-pulse preamble. This increases observability of multipath as discussed in [13], so any conclusions using this analysis are again conservative for the half-pulse amplitude method of measuring TOA. The authors in [35] presented multipath errors as a function of multipath delay which they derived from collected 1090ES data and a simulated multipath signal at $-3 \mathrm{~dB}$ signal. The resulting maximum error for multipath was around $27 \mathrm{~m}$, which highlights the benefits of a narrower pulse for multipath mitigation. To find a corresponding $2 \sigma$ value, we can use the same ratio of the DME values to get a crude error value for the Mode $\mathrm{S}$ (and Mode N) signal. Since the DME ratio for $80 \mathrm{~m}$ max error to $40 \mathrm{~m}(2 \sigma)$ is 2 , a Mode $\mathrm{S}$ error value computes as $14 \mathrm{~m}(2 \sigma)$. For Mode $\mathrm{N}$, the multipath component of the error model follows from Mode $\mathrm{S}$ as $7 \mathrm{~m}, 1 \sigma$.

\section{Time Synchronization}

The final error component to estimate for Mode $\mathrm{N}$ is that of the synchronization of the ground stations. The TDOA measurements will contain an error corresponding to the degree to which the Mode $\mathrm{N}$ ground stations are not synchronized. As Mode $\mathrm{N}$ is still only a proposed concept, the UAT signal will be the starting point to estimate an error. The UAT-based range measurements in [36] showed a bias from two ground stations which was attributed to the timing accuracy of the ground stations. As the UAT synchronization to UTC is only required to be within $500 \mathrm{~ns}(150 \mathrm{~m})$, the biases of 76 and $138 \mathrm{~m}$ were deemed to be in specification. As UAT is not intended for navigation, these tolerances are not indicative of a system such as Mode $\mathrm{N}$ that is designed primarily for navigation. Instead, an expected ground station synchronization to within $50 \mathrm{~ns}(15 \mathrm{~m})$ is assumed based on the differentially-corrected capability of eLoran [37] - which is a low-frequency system designed for navigation - and on the desired tolerance expressed in [32]. This $15 \mathrm{~m}$ maximum bias is treated as a $2 \sigma$ error component in the Mode N error model.

\section{Predicted Mode $N$ accuracy and comparison}

The results of the above analysis are summarized in Table 2. 
Table 2 - Predicted Mode N range accuracy

\begin{tabular}{cc} 
Error $(1 \sigma)$ & Value $(\mathrm{m})$ \\
\hline TOA determination (noise) & 7.5 \\
Multipath & 7 \\
Ground station synch. & 7.5 \\
\hline $\boldsymbol{\sigma}_{\text {range }}$ & $\mathbf{1 3}$
\end{tabular}

As a final check, the modeled Mode $\mathrm{N}$ accuracy will be compared to various analytical and empirical range accuracies of DME/UAT/1090ES signals. It is expected that Mode N has a better range accuracy than DME, with UAT and 1090ES being closer in performance.

A total $2 \sigma$ error for current DME performance of $92 \mathrm{~m}$ is given in [15] partially based on the data generated in [8], which translates to $46 \mathrm{~m}$ of range accuracy after dividing by two (since DME signal is two-way range). A substantial part of this error derives from the avionics bias, which in [9] is minimized for a "potential" DME error budget due to an assumed improved avionics performance. This results in a DME range $2 \sigma$ error of $34 \mathrm{~m}$; this value is chosen to compare as the effect of avionics has less of an impact in a passive ranging system such as Mode $\mathrm{N}$.

Range performance for UAT signals was evaluated in [32] with measurements showing $20 \mathrm{~m}(1 \sigma)$ error when compared to GNSS truth, not including large biases attributed to ground station synchronization and/or processing errors. The 1090ES signals do not have an inherent ranging capability, so the TDOA measurement error of two ground station signals to one receiving station is difficult to measure. Instead, [35] measures the differential TOA (DTOA) of one ground station signal received by two (GPS-synchronized) receiving stations to first identify which station transmitted the signal. When compared to the true DTOA based on ground station and receiving station coordinates, the measurements contained small biases around $10 \mathrm{~m}$ with a standard deviation also less than $10 \mathrm{~m}$. Being DTOA measurements, these do not contain ground station synchronization errors, so the reported standard deviations correspond mostly with propagation and determining TOA. The $10 \mathrm{~m}$ DTOA $1 \sigma$ error can still be converted to a range error using (4), resulting in $14 \mathrm{~m}(2 \sigma)$.

Table 3 - Comparison of Mode N with other APNT signals

\begin{tabular}{|c|c|l|}
\hline Signal & Range accuracy $(2 \sigma)$ & \multicolumn{1}{|c|}{ Notes } \\
\hline Mode N (estimated) & $26 \mathrm{~m}$ & \multicolumn{1}{|c|}{} \\
\hline DME & $34 \mathrm{~m}$ & Assuming improved DME avionics. \\
\hline UAT (from RS) & $40 \mathrm{~m}$ & Large biases not included in value. \\
\hline 1090ES (from RS) & $14 \mathrm{~m}$ & $\begin{array}{l}\text { Does not include ground station } \\
\text { synchronization errors. }\end{array}$ \\
\hline Mode S (standards) & $30 \mathrm{~m}$ & Used in ACAS avoidance logic [30]. \\
\hline
\end{tabular}

Overall, the estimated Mode $\mathrm{N}$ accuracy falls within the spectrum of empirical and analytical ranging performances from the DME, UAT, and 1090ES signals and is very close to the Mode S range accuracy published in standards such as [30]. Considering the Mode $\mathrm{N}$ signal blends aspects from all of these signals, this is a reassuring result.

\section{Coverage Assessment}

With the estimated ranging accuracy, a preliminary coverage over Germany can now be assessed. Recall that the use of existing CNS sites is just a baseline assessment, the potential Mode N layout is currently being studied by DFS. The remaining task is to provide a radius of coverage within which a ground station is available to an aircraft. This paper assumes that the Mode $\mathrm{N}$ ground stations will be able to provide a coverage radius greater than $100 \mathrm{nmi}$, which is the required coverage radius $1000 \mathrm{~W}$ DME transponders provide for aircraft above 14,500 ft [38]. Using the current surveillance site locations $(\mathrm{N}=29)$ in Germany and assuming that a minimum of three stations is necessary for positioning, the estimated position accuracy using (1) is shown in Figure 3 below. 


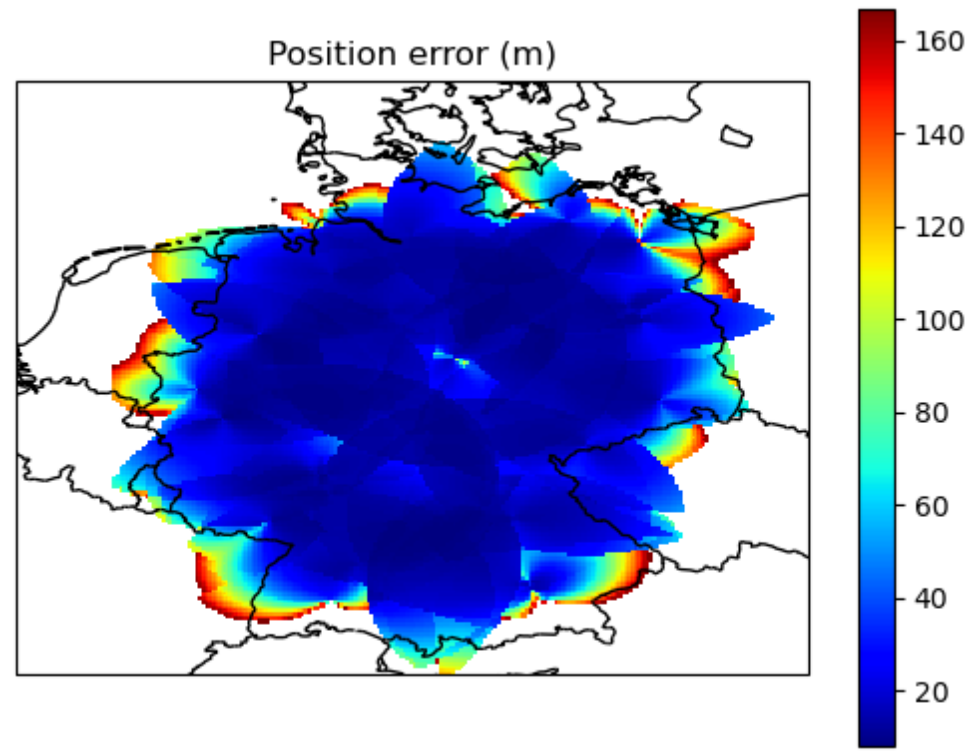

Figure 3 - Estimated position error $(\mathrm{m})$ for aircraft within $100 \mathrm{nmi}$ coverage radius using existing surveillance sites as installation locations for Mode $\mathrm{N}$ ground stations

The existing surveillance ground network was used as a baseline as there are less surveillance ground stations than DME transponders, and Mode N shares more similarities with the surveillance signals. Because Mode N would need to install new ground stations, using fewer stations incurs a lower cost depending on the amount of time beacons that would be necessary to maintain time synchronization via RF line-of-sight. The coverage assessment used a flat Germany model with the estimated range accuracy from the preceding section $(13 \mathrm{~m}, 1 \sigma)$. Atmospheric and terrain considerations were not applied in the assessment. It is important to note that this level of coverage would degrade at lower altitudes.

To determine if this level of accuracy is sufficient for the airspace modernization efforts in Europe, the desired Required Navigation Performance (RNP) accuracy requirement must be examined. For RNP 1.0, where 1.0 refers to the required 95\% or $2 \sigma$ total system error (TSE) accuracy in nautical miles, the position error allocation is assumed to be $30 \%$ of the RNP/TSE value [39]. The required position accuracy is shown below.

Table 4 - RNP required horizontal position accuracy

\begin{tabular}{|l|l|l|}
\hline RNP designation & TSE, 95\% $(2 \sigma)$ & Position Error $(1 \sigma)$ \\
\hline 1.0 & $1.0 \mathrm{nmi}, 1852 \mathrm{~m}$ & $0.3 \mathrm{nmi}, 556 \mathrm{~m}$ \\
\hline 0.3 & $0.3 \mathrm{nmi}, 556 \mathrm{~m}$ & $0.09 \mathrm{nmi}, 167 \mathrm{~m}$ \\
\hline
\end{tabular}

From Figure 3, aircraft at altitudes within the service volume supported by a 100 nmi coverage radius are capable of meeting the accuracy requirement for RNP 1.0 and 0.3 within the majority of Germany. Coverage along the border is unavailable as only German surveillance site locations were used.

\section{CONCLUSIONS}

Although the derivation of accuracy and the coverage assessment method made several simplifying assumptions, the results indicate that Mode $\mathrm{N}$ has the potential to be a feasible APNT system. To be a part of the modern airspace navigation infrastructure, additional requirements to accuracy must also be met. The integrity requirement is harder to meet than accuracy, and requires either redundant information available to the aircraft for a RAIM-like algorithm or a ground-based monitoring/augmentation system. Perhaps the biggest challenge to implementing the Mode $\mathrm{N}$ infrastructure is maintaining an RF-based time synchronization network. The design outlined in [1] called for additional time beacons when using existing DME sites for the installation of Mode N stations; as there are more DME stations than surveillance, the coverage assessment shown here would not be accurate without additional time beacons to maintain synchronization within 50 ns. The TDMA approach to mitigating intersystem interference might require additional management if more ground stations are needed and 
if, as noted in [36], a higher update rate than the planned $1 \mathrm{~Hz}$ is desired. Convincing aircraft operators to update their avionics is another challenge to Mode $\mathrm{N}$ implementation, although the inclusion of DME functionality in the Mode N avionics seeks to ease that transition.

\section{DISCLAIMERS}

The views expressed herein are those of the authors and are not to be construed as official or reflecting the views of Deutsche Flugsicherung.

\section{REFERENCES}

[1] Deutsche Flugsicherung, "Basic Design Document (Version 2.0) for Mode N: A new System Concept of Navigation \& A-PNT for Aviation," 2020.

[2] S. Marquard, "Mode N - A Concept of Modern Navigation and an Alternative Position, Navigation \& Timing System for Aviation," Innovation im Fokus, pp. 23-28, February 2017.

[3] John A. Volpe National Transportation Systems Center, "Vulnerability Assessment of the Transportation Infrastructure Relying on the Global Positioning System," 2001.

[4] M. J. Narins, L. Eldredge, P. K. Enge, M. Harrison, R. Kenagy and S. C. Lo, "Alternative Positioning Navigation and Timing -- The Need for Robust Radionavigation," in Proceedings of the Royal Institute of Navigation, London, UK, 2010.

[5] P. Enge, E. Swanson, R. Mullin, K. Ganther, A. Bommarito and R. Kelly, "Terrestrial Radionavigation Technologies," Navigation, Journal of the Institute of Navigation, vol. 42, no. 1, pp. 61-108, 1995.

[6] R. Lilley and R. Erikson, "DME/DME for Alternate Position, Navigation, and Timing (APNT)," [White Paper] Federal Aviation Administration, 2012.

[7] E. Kim, "Investigation of APNT optimized DME/DME network using current state-of-the-art DMEs: Ground station network, accuracy, and capacity," in Proceedings of the 2012 IEEE/ION Position, Location and Navigation Symposium, Myrtle Beach, SC, 2012.

[8] M. Harris, T. Murphy, R. Lilley and R. Erikson, "Performance of Current Distance Measuring Equipment and Implications on Alternative Position Navigation and Timing for Aviation," in Proceedings of the 25th International Technical Meeting of the Satellite Division of the Institute of Navigation (ION GNSS 2012), Nashville, TN, 2012.

[9] S. Lo, Y. H. Chen, P. Enge, B. Peterson and R. Erikson, "Distance Measuring Equipment Accuracy Performance Today and for Future Alternative Position Navigation and Timing (APNT)," in Proceedings of the 26th International Techinical Meeting of the Satellite Division of The Institute of Navigation (ION GNSS+ 2013), Nashville, TN, 2013.

[10] S. Lo and P. Enge, "Capacity Study of Two Potential Alternative Position Navigation and Timing (APNT) Services for Aviation," in Proceedings of the 2011 International Technical Meeting of The Institute of Navigation, San Diego, CA, 2011.

[11] S. Lo and P. Enge, "Assessing the Capability of DIstance Measuring Equipment (DME) to Support Future Air Traffic Capacity," NAVIGATION, Journal of the Institute of Navigation, vol. 59, no. 4, pp. 249-261, 2012.

[12] S. Lo, P. Enge, F. Niles, R. Loh, L. Eldredge and M. Narins, "Preliminary Assessment of Alternative Navigation Means for Civil Aviation," in Proceedings of the 2010 International Technical Meeting of The Institue of Navigation, San Diego, CA, 2010.

[13] K. Li and W. Pelgrum, "Optimal Time-of-Arrival estimation for Enhanced DME," in Proceedings of the 24th International Technical Meeting of the Satellite Division of The Insititute of Navigation (ION GNSS 2011), Portland, OR, 2011.

[14] S. Lo, Y. H. Chen, B. Segal, B. Peterson, P. Enge, R. Erikson and R. Lilley, "Containing a Difficult Target: Techniques for Mitigating DME Multipath to Alternative Position Navigation and Timing (APNT)," in Proceedings of the 2014 International Technical Meeting of The Institute of Navigation, San Diego, CA, 2014.

[15] E. Kim, "Improving DME Peformance for APNT Using Alternative Pulse and Multipath Mitigation," IEEE Transactions on Aerospace and Electronic Systems, vol. 53, no. 2, pp. 877-887, 2017.

[16] V. Gordo, G. Paz and J. San-Juan, "DME Multipath Simulations as a Critical enabler to Support DME/DME RNP Reversion". 
[17] W. Pelgrum, K. Li, M. Smearchek and F. van Graas, "eDME Architecture Development and Flight-Test Evaluation," in Proceedings of the 25th International Technical Meeting of the Satellite Division of The Institute of Navigation (ION GNSS 2012), Nashville, TN, 2012.

[18] L. Eldredge, P. Enge, M. Harrison, R. Kenagy, S. Lo, R. Loh, R. Lilly, M. Narins and R. Niles, "Alternative Positioning, Navigation \& Timing (PNT) Study," Paper presented at the working group meeting of the International Civil Aviation Organisation Navigation Systems Panel (NSP), Montreal, Canada, 2010.

[19] S. Lo, B. Peterson, D. Akos, M. Narins, R. Loh and P. Enge, "Alternative Position Navigation \& Timing (APNT) Based on Existing DME and UAT Ground Signals," in Proceedings of the 24th International Technical Meeting of the Satellite Division of The Insitute of Navigation (ION GNSS 2011), Portland, OR, 2011.

[20] S. Lo and P. Enge, "Signal Structure Study for a Passive Ranging System using Existing Distance Measuring Equipment (DME)," in Proceedings of the 2012 Iternational Technical Meeting of The Institute of Navigation, Newport Beach, CA, 2012.

[21] S. Lo, P. Enge and M. Narins, "Design of a Passive Ranging System Using Existing Distance Measuring Equipment (DME) Signals \& Transmitters," NAVIGATION, Journal of the Institute of Navigation, vol. 62, no. 2, pp. 131-149, 2015.

[22] S. Lo, Y.-H. Chen, P. Enge, W. Pelgrum, K. Li, G. Weida and A. Soelter, "Flight test of a pseudo-ranging signal compatible with existing distance measuring equipment (DME) ground stations," NAVIGATION, Journal of the Institute of Navigation, vol. 67, no. 3, pp. 567-581, 2020.

[23] D. Lawrence, "FAA Navigation Programs Update," in Presented to Civil GPS Service Interface Committee, Sep. 2020.

[24] Project Team Terrestrial participants of ICAO Communications Panel, "LDACS White Paper - A Roll-out Scenario," Montreal, Canada, 2019.

[25] O. Osechas, S. Narayanan, O. Garcia Grespillo, G. Zampieri, G. Battista, R. Kumar, N. Schneckenburger, E. Lay, B. Belabbas and M. Meurer, "Feasibility Demonstration of Terrestrial RNP with LDACS," in Proceedings of the 32nd International Technical Meeting of the Satellite Division of The Institute of Navigation (ION GNSS+ 2019), Miami, FL, 2019.

[26] P. D. Groves, "Principles of Radio Positioning," in Principles of GNSS, Inertial, and Multisensor Integrated Navigation Systems, 2nd ed., Boston | London, Artech House, 2013, pp. 255-298.

[27] S. Lo, Y.-H. Chen, A. Barrows, A. Perkins, T. Reid, P. Enge and S.-S. Jan, "Using Traffic Information Services Broadcast (TIS-B) Signals for Aviation Navigation," in Proceedings of IEEE/ION PLANS 2016, Savannah, GA, 2016.

[28] D. Lawrence, "Alternative Positioning, Navigation, and Timing (APNT)," in 13th National Space-based PNT Advisory Board, 2014.

[29] M. C. Stevens, Secondary Surveillance Radar, Norwood, MA: Artech House, Inc., 1988.

[30] Aeronautical Telecommunications, Annex 10 to the Convention on International Civil Aviation, 5th ed., Vols. IV, Surveillance and Collision Avoidance Systems, ICAO, 2014.

[31] J. Wollweber, R. Mallwitz and S. Marquard, "Proposal for a frequency flexible SSR/Mode N system," TE im Fokus Information aus dem Bereich Forschung und Entwicklung der DFS Deutsche Flugsicherung GmbH, pp. 26-30, 2012.

[32] S. Lo, Y. H. Chen, P. Enge and M. Narins, "Techniques to Provide Resilient Alternative Positioning, Nvigation, and Timing (APNT) Using Automatic Dependent Surveillance - Broadcast (ADS-B) Ground Stations," in Proceedings of the 2015 International Technical Meeting of The Institute of Navigation, Dana Point, CA, 2015.

[33] S. Lo, Y.-H. Chen, S. Zhang and P. Enge, "Hybrid APNT: Terrestrial Radionavigation to Support Future Aviation Needs," in Proceedings of the 27th International Technical Meeting of the Satellite Division of the Institute of Navigation (ION GNSS+ 2014), Tampa, Florida, 2014.

[34] P. Misra and P. Enge, Global Positioning System: Signals, Measurements, and Performance, rev. 2nd ed., Lincoln, MA: Ganga-Jamuna Press, 2001.

[35] Y. H. Chen, S. Lo, P. Enge and S. S. Jan, "Evaluation \& Comparison of Ranging Using Universal Access Transceiver (UAT) and $1090 \mathrm{MHz}$ Mode S Extended Squitter (Mode S ES)," in Proceedings of IEEE/ION PLANS 2014, Monterey, CA, 2014.

[36] Y.-H. Chen, S. Lo, S.-S. Jan, G.-J. Liou, D. M. Akos and P. Enge, "Design and Test of Algorithms and Real-Time Receiver to use Universal Acces Transceiver (UAT) for Alternative Positioning Navigation and Timing (APNT)," in Proceedings of the 27th International Technical Meeting of the Satellite Division of the Institute of Navigation (ION GNSS+ 2014), Tampa, FL, 2014. 
[37] G. Offermans, E. Jahannessen, C. Schue, J. Hirschauer and E. Powers, "UTC Synchronization and Stratum-1 Frequency Recovery Using eLoran: The Alternate Basket for Your Eggs," in Proceedings of the 45th Annual Precise Time and Time Interval Systems and Applications Meeting, Bellevue, WA, 2013.

[38] Federal Aviation Administration, "FAA-E-2996 Performance Specification Distance Measuring Equipment (DME)," 2008.

[39] The European Organisation for Civil Aviation Equipment, "Minimum Aviation System Performance Standards: Requried Navigation Performance for Area Navigation," 2014. 